Check for updates

Cite this: RSC Adv., 2019, 9, 15042

Received 23rd February 2019

Accepted 7th May 2019

DOI: 10.1039/c9ra01381d

rsc.li/rsc-advances

\section{Construction of novel multifunctional luminescent nanoparticles based on DNA bridging and their inhibitory effect on tumor growth $\dagger$}

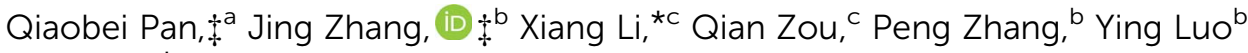 \\ and Yi Jin*a
}

Cyclic RGD peptide was introduced onto the surface of silver nanoparticle (AgNP)-single strand DNA (ssDNA)-graphene quantum dots (GQDs) (ADG) after coating with a hybrid phospholipid material (ADGDDPC) to be used for antitumor treatment. The Ag and ssDNA content was quantified. The morphology and properties of the nanoparticles were characterized by ultraviolet-visible absorption spectroscopy (UV-VIS), scanning electron microscopy (SEM), transmission electron microscopy (TEM), and atomic force microscopy (AFM). The etching effect of $\mathrm{H}_{2} \mathrm{O}_{2}$ on the AgNPs and the cleavage of DNA was observed. The cytotoxicity of the ADG-DDPC was investigated using the cell viability and LDH content. The cell uptake was evaluated by using the fluorescence recovery of the GQDs in the ADG-DDPC. The antitumor effects of ADG-DDPC were also evaluated. The content of the ssDNA was $15.3 \mu \mathrm{gL}^{-1}$. The content of the silver element in AgNPs was $3.75 \mu \mathrm{g} \mathrm{mL}^{-1}$ and $20.43 \mu \mathrm{g} \mathrm{mL}^{-1}$ in ADG-DDPC. ADG were distributed uniformly with the GQDs on the surface. After coating with hybrid phospholipid membranes containing DSPE-PEG2000-CRGD, ADG-DDPC was detected with an average size of $25.2 \mathrm{~nm}$ with a low $\mathrm{IC}_{50}$ of $209.68 \mathrm{ng} \mathrm{mL}^{-1}$ and showed LDH activity on HeLa cells. A better cellular uptake of ADG-DDPC was observed in HeLa cells, compared with CRGD-unmodified ADG nanoparticles (ADG-DDP), up to 6 and $12 \mathrm{~h}$ using the fluorescence recovery of GQDs as a measurement. Compared with ADG-DDP (3.6 mg of silver equivalent per $\mathrm{kg}$ body weight), ADG-DDPC at the same dose significantly halted $50.9 \%$ of tumor growth with little change to body weights when compared with a PTX Injection (10 $\mathrm{mg} \mathrm{kg}^{-1}$ ). The novel nanoparticles, ADG-DDPC, could target tumor sites to exhibit multifunctional inhibition on tumor growth with little toxicity.

\section{Introduction}

Cancer is a group of diseases that originate in human epithelial tissue and is the most common type of malignancy. ${ }^{\mathbf{1}}$ Chemotherapy and radiotherapy are commonly used in traditional cancer treatment. ${ }^{2,3}$ These treatments can inhibit the growth of tumors to some extent, but can also cause systemic toxicity,

${ }^{a}$ National Pharmaceutical Engineering Center for Solid Preparation in Chinese Herbal Medicine, Jiangxi University of Traditional Chinese Medicine, Nanchang, Jiangxi Province, People's Republic of China. E-mail: jinyidr@163.com; Tel: +86-791-8711-9610

${ }^{b}$ Key Laboratory of Modern Preparation of TCM, Ministry of Education, Jiangxi University of Traditional Chinese Medicine, Nanchang, Jiangxi Province, People's Republic of China

${ }^{c}$ State Key Laboratory of Innovative Drug and Efficient Energy-Saving Pharmaceutical Equipment, Jiangxi University of Traditional Chinese Medicine, Nanchang, Jiangxi Province, People's Republic of China. E-mail: 20111013@jxutcm.edu.cn; Tel: +86791-8711-9661

$\dagger$ Electronic supplementary information (ESI) available. See DOI: 10.1039/c9ra01381d

\$ These authors contributed equally to the work. resistance tolerance and other defects. Normal human tissue has a huge adverse reactions to these treatments, making this a difficult problem in medical history. ${ }^{4}$ Obviously, a single, traditional treatment method can no longer meet our clinical needs. Therefore, treatments based on tumor specific cell surface receptor binding peptides, such as apoptosis peptides, suicide genes, and imaging dyes have been developed. ${ }^{5-7}$ Many have been designed to achieve accurate and systematic delivery and significantly reduce the damage to normal tissues caused by drugs or tumor suppressing substances. ${ }^{8}$ In recent decades, many research groups have developed novel multi-functional preparations such as dual targeting liposomes, ${ }^{9}$ microcapsules and microspheres, ${ }^{\mathbf{1 0}, 11}$ inclusion complexes, ${ }^{\mathbf{1 2}}$ and nanoparticles. ${ }^{\mathbf{1 3 , 1 4}}$

Graphene quantum dots (GQDs) are a new type of materials that have been evolved from graphene. The most obvious difference between GQDs and graphene is that the energy level band gap of GQDs is about $7 \mathrm{eV}$, compared to the energy band gap of graphene which is zero. This difference gives GQDs have a stronger edge effect and a smaller size effect, and the fluorescence quantum yield is significantly higher than that of 
graphene. ${ }^{15,16}$ GQDs are generally about $10 \mathrm{~nm}$ in size, and not only retain the properties of graphene, that is, the large $\pi$ bond, but also introduces many characteristic light energy clusters during the synthesis, such as hydroxyl, carboxyl, amino, and so forth. ${ }^{17}$ This makes it possible to provide a theoretical basis for the successful connection to other materials. Owing to their good biocompatibility, chemical inertness and stable photoluminescence, GQDs have broad prospects for applications in biosensor imaging and optoelectronic devices. By using the photoluminescence properties of GQDs and changing their particle size of GQDs, the gap between the conduction band and the valence band can be changed, which directly affects the variation of the emission wavelength of GQDs with the change of the particle size. ${ }^{18}$ At present, a large number of published studies have reported the synthesis of GQDs, and have shown that different fluorescence colors can be generated. ${ }^{19-22}$ If we can accurately transport GQDs to the tumor site and make use of the important photoluminescence characteristics under the action of an external light source, by focusing the light at the tumor site, we could overcome the problem of the tumor site being difficult to determine and could reach a the radical cure..$^{23-25}$

Silver nanoparticles (AgNPs) have been developed for a broader range of applications, such as for use as photosensitive materials, ${ }^{26}$ decorative materials, catalysis, antibacterial materials and in medicine. ${ }^{27-30}$ Many methods have been reported for the preparation of AgNPs, including spherical, tubular, rod-shaped, and so forth, and these are sized between 1-100 nm. ${ }^{31-35}$ AgNPs have a very large specific surface area and a small size effect and are often used as a fungicide. Owing to the increasing advantages of using AgNPs in the bactericidal field, many research groups have begun to study their potential in anti-tumor cells. ${ }^{36-40}$ Research teams have shown that AgNPs does have varying degrees of killing effect on many cancer cell lines, such as breast cancer cells, ${ }^{41,42}$ lymphoma cells, ${ }^{43}$ cervical cancer cells, ${ }^{44}$ and lung cancer cells,${ }^{45}$ which provided the idea of developing a novel inorganic anti-tumor nanoscale preparation. AgNPs can also indirectly induce the production of reactive oxygen species (ROS) by inducing inflammatory responses in cells. ${ }^{46-49} \mathrm{ROS}$ is a series of reactive oxygen species produced by aerobic cells in the metabolic process, including: $\mathrm{O}^{2-}, \mathrm{H}_{2} \mathrm{O}_{2}$, $\mathrm{HO}_{2}$ and $\cdot \mathrm{OH}^{50}$ Aceituno et al. indicated that the toxicity of reactive oxygen species mainly comes from the peroxidation reaction with cellular proteins and unsaturated fatty acids, which destroys the cell membrane structure and further leads to apoptosis. ${ }^{51}$

Here, we designed AgNPs/GQDs cores bridged by a single strand DNA (ssDNA). The design was developed to take advantage of the following points: (1) nano-silver demonstrates strong cytotoxicity against many cancer cells; ${ }^{52,53}$ (2) GQDs with stable photoluminescence can stimulate cells to produce reactive oxygen species (ROS), which has a certain inhibitory effect on cell growth; (3) ssDNA, is connected to the AgNPs/GQDs by $\pi-\pi$ stacking between the GQDs and the surface of AgNPs-DNA. ${ }^{54}$

Therefore, cRGD cyclic peptides, that have the sequence: cyclo (Arg-Gly-Asp-D-Phe-Cys), were used to modify hybrid phospholipids membrane after coating ADG cores to achieve long-circulation profile and tumor targeting. ${ }^{55}$ First, the RGD specifically acts on the surface of the tumor cells or the surface of the $\alpha_{v} \beta_{3}$ integrin receptor on the tumor vascular endothelial cells. ${ }^{56}$ Then the cell surface specific proteolytic enzyme cleaves the cRGD peptide to form an activated CendR peptide which can specifically recognizes and activates the neuropilin-1 receptor on the cell surface and can therefore achieve targeted delivery through the cell membrane. ${ }^{57}$ We used cRGD as a specific transport carrier to externally modify the longcirculation time of the nanomaterials. The coupled nanomaterials can not only maintain long cycle properties in vivo, but the delivery of nanomaterials to the tumor during this process is also maximized. ${ }^{58-61}$

The aim of this article is to describe the construction of a new nano-formulation which can target the tumor tissue and achieve the long-circulation of nanoparticles in the body by fabricating an inorganic nanosilver material as the internal core and connecting this, using an ssDNA, to GQDs, which have a fluorescence localization function.

\section{Experimental section}

\section{Materials}

An ssDNA labeled with sulfhydryl, SH-DNA (5'SHGGAGTTGGGGAGGAGG- $3^{\prime}$ ), was purchased from Sangon Biotech Co., Ltd (Shanghai, China). The GQDs (XF093) were purchased from Nanjing XF NANO Materials Tech (Nanjing, China). Tris(2-carboxyethyl)phosphine (TCEP) was purchased from Sigma-Aldrich (Saint Louis, MD, USA). 1,2-Dioleoyl-3trimethylammonium-propane chloride salt (DOTAP) and 1,2distearoyl-sn-glycero-3-phosphoethanolamine- $N$-[methoxy(polyethylene glycol)-2000] (DSPE-PEG ${ }_{2000}$ ) were purchased from Shanghai Advanced Vehicle Technology Pharmaceutical Ltd. (Shanghai, China). 1,2-Distearoyl-sn-glycero-3-phosphoethanolamine- $N$-[methoxy(polyethylene glycol)-2000]-cRGD (DSPE$\mathrm{PEG}_{2000}$-cRGD) was from Xi'an Ruixi Biological Technology Co., Ltd. (Xi'an China). The ssDNA Quantitation Kit was purchased from Invitrogen (Thermo Fisher Scientific). Paclitaxel (PTX) Injection was obtained from Jiangsu Aosaikang Pharmaceutical CO., Ltd (5 mL: $30 \mathrm{mg}$, CFDA approval number H20064300, Jiangsu, China). Silver standard storage solution (Ag, GSB 041712-2004, uniquely identifies: 17A039-1, $1000 \mathrm{mg} \mathrm{L}^{-1}$ ) was obtained from National Nonferrous Metals and Electronic Materials Analysis and Testing Center. Rh internal standard element $\left(100 \mathrm{mg} \mathrm{L}^{-1}\right)$ was purchased from Thermo Fisher Scientific Co., Ltd. 50 nm filter (85037-547-44) were purchased from Sartorius CO., Ltd.

The human cervical cancer cell line HeLa was obtained from the Cell Bank of the Chinese Academy of Sciences (Shanghai, China). HeLa cells were cultured in DMEM (Life Technologies, Carlsbad, CA, USA) containing 10\% fetal bovine serum (Life Technologies), L-glutamine (2 $\mathrm{mM})$, and penicillin-streptomycin solution ( $40 \mathrm{U} \mathrm{mL}^{-1}$-each; Life Technologies). Cells were cultivated in a humidified incubator at $37{ }^{\circ} \mathrm{C}$ and under $5 \%$ $\mathrm{CO}_{2}$. The cells were harvested with $0.05 \%$ trypsin-EDTA before subculture. The cell counting kit-8 (CCK-8) was purchased from Thermo Fisher Scientific (Waltham, MA, USA). The lactate 
dehydrogenase (LDH) kit was purchased from Beijing solarbio science \& technology Co., Ltd (Beijing, China).

The animal experiments were carried out in accordance with the Guide for the Care and Use of Laboratory Animals (National Research Council of China, Beijing, China). The study protocol was approved by the Institutional Animal Care and Use Committee of Jiangxi University of Traditional Chinese Medicine (Nanchang, China). Female BALB/c nude mice (6 weeks; 18-20 g) were provided by Hunan SJA Laboratory Animals (no: SCXK [XIANG] 2016-0002, Hunan, China). Animals were cared for in the animal laboratory center of Jiangxi University of Traditional Chinese Medicine. The animal room was well ventilated and had a regular $12 \mathrm{~h}$ light dark cycle throughout the experimental period.

\section{Synthesis of silver nanoparticles (AgNPs)}

In this paper, the chemical reduction method and sodium borohydride were used to deoxidize silver atoms to give AgNPs in aqueous solution. After preliminary experiments, it was determined that $30 \mathrm{~mL}$ of $3.3 \mathrm{mM}$ sodium citrate aqueous solution and $90 \mathrm{~mL}$ of $6.6 \mathrm{mM}$ sodium borohydride aqueous solution should be taken and mixed evenly under ice bath conditions. Then, $3.3 \mathrm{mM}$ silver nitrate drops were added to the mixture, and the ice bath and stirring conditions were maintained during the dropping process. After the reaction was complete, the solution was placed in the dark overnight, to ensure that the sodium borohydride was completely decomposed. After aging, the mixture was dialyzed against a dialysis bag with a molecular weight cut off of $3500 \mathrm{Da}$, and the dialysate was changed every half hour for at least $6 \mathrm{~h}$. The purpose of the dialysis was to remove some of the ions from the outside of the silver nanoparticles. After dialysis, the silver nanoparticle solution in the dialysis bag was collected for further experiments.

\section{Synthesis of ADG cores coating hybrid lipid membrane (ADG- DDPC)}

The scheme for the synthesis of the ADG cores coated with a hybrid lipid membrane (ADG-DDPC) is shown in Fig. 1.

First, $20 \mu \mathrm{L}$ of $10 \mathrm{mM}$ TCEP and $20 \mu \mathrm{L}$ of $100 \mu \mathrm{M}$ SH-DNA were incubated for $1 \mathrm{~h}$ in a Britton-Robinson buffer (BR buffer, $\mathrm{pH}=6)$. Then, $20 \mu \mathrm{L}$ of SH-DNA $(50 \mu \mathrm{M})$ was added to $6 \mathrm{~mL}$ of the AgNPs solution (the $\mathrm{pH}$ of the AgNPs solution was

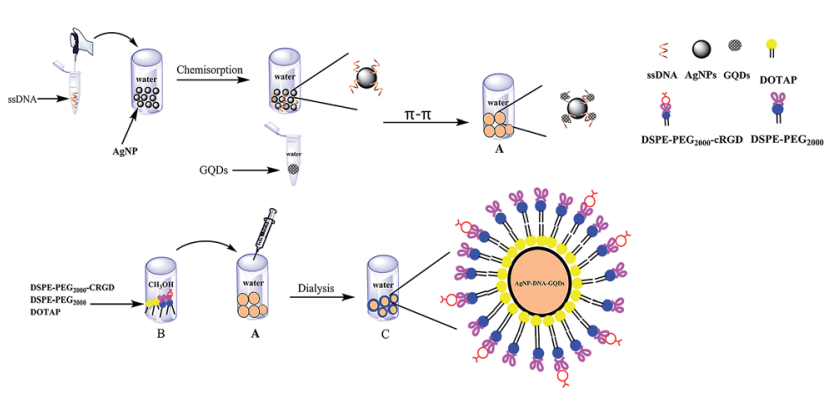

Fig. 1 Preparation process of ADG-DDP or ADG-DDPC. adjusted to neutral by adding $1.5 \mathrm{~mL}$ of BR buffer). The solution was incubated for about 5 min to allow the adsorption of DNA onto the AgNPs. After this, a citrate buffer ( $500 \mathrm{mM}, \mathrm{pH}=3$ ) was added into the AgNPs solution to reach a final concentration of $5 \mathrm{mM}$. After $5 \mathrm{~min}$ incubation, the same amount of citrate buffer was added into the solution again to achieve a final concentration of $10 \mathrm{mM}$ citrate. After incubating for $15 \mathrm{~min}$, the excess DNA was removed by centrifuging at $12000 \mathrm{rpm}$ for $30 \mathrm{~min}$ and the remaining pellet was then washed three times with BR buffer. The prepared AgNP-DNA conjugates were dispersed in BR buffer for further use. Then, the AgNP-DNA was re-dispersed in BR buffer and a final concentration of $50 \mu \mathrm{g} \mathrm{mL}{ }^{-1}$ GQDs was added. The solution was incubated at room temperature for $2 \mathrm{~h}$ to obtain ADG cores. After dialysis for $6 \mathrm{~h}$ in a dialysis bag with a molecular weight cut off of $10000 \mathrm{Da}$, the non-adsorbed GQDS were removed and used.

A hybrid phospholipids solution $(200 \mu \mathrm{L})$ containing $6.87 \mathrm{mM}$ DOTAP and $0.38 \mathrm{mM}$ DSPE-PEG 2000 -cRGD in methanol was slowly injected into the prepared ADG cores and incubated for about $30 \mathrm{~min}$ at $40{ }^{\circ} \mathrm{C}$ in a water bath with gentle shaking. After the phospholipids were adsorbed onto the surface of the ADG cores, $2 \mathrm{~mL}$ of the obtained suspension were dialyzed for more than $8 \mathrm{~h}$ in the dialysate tube to ensure that the organic phase methanol was completely dialyzed. The prepared phospholipid nanoparticles were filtered through a $50 \mathrm{~nm}$ filter to obtain ADG-DDPC. The ADG cores coated with the hybrid lipid membrane containing DOTAP and DSPE-PEG 2000 (ADG-DDP) were prepared using almost the same protocol as that of the ADG-DDPC, except that $0.43 \mathrm{mM}$ of DSPE-PEG 2000 was used to replace the DSPE-PEG 2000 -cRGD.

\section{Characterization of ADG-DDPC}

Several methods were used to characterize the preparation of ADG-DDPC.

Determination of the ssDNA adsorbed by the AgNPs was performed by using an ssDNA quantitation kit Quant-iTTM OliGreen ${ }^{\circledR}$ SSDNA Kit. After the DNA was adsorbed onto the surface of AgNPs, a three-fold volume of BR buffer $(\mathrm{pH}=3)$ was added, centrifuged at $12000 \mathrm{rpm}$ for $20 \mathrm{~min}$, washed five times using BR buffer, and the total SsDNA in the supernatant was measured as recommended by manufacturer.

The concentration of silver nanoparticles was measured using an inductively coupled plasma mass spectrometer (ICPMS), (Thermo Fisher Scientific). The ICP-MS was performed using the following conditions: RF power $1550 \mathrm{~W}$, cooling gas $14 \mathrm{~L} \mathrm{~min}^{-1}$, auxiliary gas $0.7763 \mathrm{~L} \mathrm{~min}^{-1}$, atomization gas $1.0749 \mathrm{~L} \mathrm{~min}^{-1}$, spray chamber temperature $2.5{ }^{\circ} \mathrm{C}$, and peristaltic pump speed $40 \mathrm{rpm}$.

The etching of the $\mathrm{Ag}$ in the ADG cores under $\mathrm{H}_{2} \mathrm{O}_{2}$ was performed by adding $200 \mu \mathrm{M} \mathrm{H}_{2} \mathrm{O}_{2}$ into $400 \mu \mathrm{L}$ of BR buffer containing $180 \mathrm{pM}$ of the ADG cores. The mixture was reacted for $40 \mathrm{~min}$ at $37^{\circ} \mathrm{C}$. Then the morphology of the ADG after $\mathrm{H}_{2} \mathrm{O}_{2}$ addition was observed using transmission electron microscopy (TEM).

Ultraviolet-visible absorption spectroscopy (UV-VIS) (UV2550 spectrophotometer, Shimadzu, Japan) was used to obtain 
the spectroscopic characterization of ADG-DDPC. The structural characterizations of ADG-DDPC was confirmed by using a FEI Quanta 250 scanning electron microscope (SEM) (Thermo Fisher Scientific, USA), a JEM2100 electron microscopy (TEM) (JEOL Ltd., Japan) and an atomic force microscopy (AFM) images were acquired under ambient conditions using a SPM9700 AFM (Shimadzu, Japan) in the tapping mode. The particle size distributions were determined using the Nano ZS ZEN3690 (Malvern Instruments Ltd., Malvern, UK). Fluorescence detection of $\mathrm{H}_{2} \mathrm{O}_{2}$ were determined using the FLS980 Steady-state transient fluorescence spectrometer (Edinburgh instruments co., Ltd.)

\section{Cell viability}

The cytotoxicity of the AgNPs, GQDs, ADG-DDP and ADG-DDPC complexes against HeLa cells were assessed using the CCK-8 method. HeLa cells were seeded in 96-well plates at a density of $5 \times 10^{3}$ cells per well and incubated for $24 \mathrm{~h}$ at $37^{\circ} \mathrm{C}$ in $5 \%$ $\mathrm{CO}_{2}$. Then the culture medium was removed and fresh medium containing AgNPs, GQDs, ADG-DDP and ADG-DDPC complexes were added to each well. After $24 \mathrm{~h}$ of incubation, $10 \mu \mathrm{L}$ of the CCK-8 solution was added and further incubated for $1 \mathrm{~h}$. The optical density of the solution was measured at $450 \mathrm{~nm}$ using a Spectra-Max M3 microplate reader (Molecular Devices, CA, USA). The cell viability for each of the treated groups was calculated and normalized to control untreated cells. Each concentration was detected using six wells. The mean drug concentration for $50 \%$ inhibition of cell growth $\left(\mathrm{IC}_{50}\right)$ was calculated.

\section{LDH activity}

According to the instructions on the kit, the catalytic production of $1 \mathrm{nM}$ pyruvate per minute $1 \times 10^{4}$ cells or bacteria at $37^{\circ} \mathrm{C}$ was defined as an enzyme activity unit. HeLa cells were seed into the 6-well plates at a density of $5 \times 10^{5}$ cells per well and incubated for $24 \mathrm{~h}$ at $37^{\circ} \mathrm{C}$ in $5 \% \mathrm{CO}_{2}$. Cells were divided into the treated group, the control group and the standard group, and each group was set with three replicate wells at each concentration. The cells were collected into a centrifuge tube the supernatant was discarded after centrifugation, and the cells were disrupted using an ultrasonicator (ice bath, power $20 \%$, sonication for $3 \mathrm{~s}$, interval $10 \mathrm{~s}$, repeated 30 times); $8000 \mathrm{~g}$ centrifuge for $10 \mathrm{~min}$ at $4{ }^{\circ} \mathrm{C}$, the supernatant was then removed and placed on ice prior to being measured. The LDH activity in each group was obtained as recommended by the manufacturer.

\section{Cell uptake}

HeLa cells were incubated with $24 \mathrm{ng} \mathrm{mL}^{-1}$ of ADG-DDP and ADG-DDPC for $6,12 \mathrm{~h}$ in a laser confocal dish. After the incubation, the cells were washed and fixed with $4 \%$ paraformaldehyde. Finally, the cells were washed three times. The intracellular localization of ADG-DDP or ADG-DDPC was visualized using an LSM 710 confocal microscope from Zeiss (Oberkochen, Germany). The fluorescence signals of the GQDs were excited at $350 \mathrm{~nm}$ and detected at $425 \mathrm{~nm}$, respectively.
The percentages of fluorescence positive cells (fluorescence caused by released GQDs) were quantified by Image J software.

\section{In vivo antitumor activity}

The in vivo antitumor activity was assessed in HeLa xenograft mice. The HeLa cells $\left(1 \times 10^{7} /\right.$ per mouse $)$ were injected subcutaneously into the right anterior armpit of BALB/c nude mice. When the tumor volume reached approximately 300-400 $\mathrm{mm}^{3}$, the tumor-bearing mice were randomly divided into 4 groups (6 mice per group) and were intravenously injected with normal saline, PTX Injection (10 $\mathrm{mg} \mathrm{kg}^{-1}$ ), ADG-DDP and ADGDDPC (3.6 mg of silver equivalent per kg body weight) every two days, three times in total. The tumor volume and body weight of the mice were recorded once every two days. The tumor volume was calculated using the following formula (1):

$$
V=\left(W^{2} \times L\right) / 2,
$$

where $V$ is the tumor volume, $W$ the smaller perpendicular diameter, and $L$ the larger perpendicular diameter. At the end of the experiments, the mice were sacrificed and the tumors were collected for measurement of the tumor weight.

\section{Tissue analysis}

The BALB/c nude mice, body weight $20-25 \mathrm{~g}$, were used for tissue analysis studies after HeLa cells were implanted intradermally into the armpit for one week. Forty tumor-bearing mice were fasted overnight and randomly divided into three groups of ten mice each. GQDs $\left(10 \mathrm{mg} \mathrm{kg}{ }^{-1}\right.$, the dose was equivalent to GQDs in the ADG-DDP and ADG-DDPC), ADG-DDP and ADG-DDPC were injected intravenously (3.6 $\mathrm{mg}$ of silver equivalent per $\mathrm{kg}$ body weight). Another ten animals were sacrificed without treatment, and their tissues were used as blank controls. The animals were decapitated in groups of three at 360 minutes, and three tumor samples per group tumor tissue were removed, and washed of residual blood, air-dried, and weighed. Tissue samples were homogenized with saline $0.2 \mathrm{mg} \mathrm{mL}^{-1}$, take $1 \mathrm{~mL}$ tissue homogenate into the microwave digestion tube and add internal standard (IS) working solution of $\mathrm{Rh}$ at $50 \mathrm{~mL}$ volumetric flask, the final concentration of IS was $50 \mu \mathrm{g} \mathrm{L}^{-1}$, then add $5 \mathrm{~mL} \mathrm{HNO}_{3}(2 \%)$ solution, digest according to the microwave heating program (temperature rise $180{ }^{\circ} \mathrm{C}$, pressure $25 \mathrm{bar}$, hold for $15 \mathrm{~min}$ ), and transfer the liquid, using the inductively coupled plasma mass spectrometer measurement as described earlier. Take 0.1, 0.5, 1, 5, 10, 20, 50 $\mu \mathrm{g} \mathrm{L}^{-1}$ of Ag standard solution and add the same volume of IS, digested as described earlier, using the inductively coupled plasma mass spectrometer measurement as described earlier.

The remaining mice were sacrificed on the tenth day to take the heart, liver, spleen, lungs, kidneys, brain, and tumor tissue of BALB/c nude mice were evaluated by histology with H\&E staining, in which hematoxylin stains nuclei to bluish-purple colors, and eosin stains cytoplasm and the stroma matrix to red-pink colors. 


\section{Statistical analyses}

All the results except in vivo data are presented as the mean \pm standard deviation (SD). The Student's $t$-test and one-way analysis of variance were used to evaluate significance. $p<$ 0.05 was considered significant.

\section{Results and discussion}

\section{Characterization of ADG-DDPC}

The amount of the ssDNA adsorbed onto the AgNPs was calculated by using an ssDNA quantitation kit (Quant-iT ${ }^{\mathrm{TM}}$ OliGreen ${ }^{\circledR}$ ssDNA Kit) as recommended by manufacturer, and was found to be $15.3 \mu \mathrm{g} \mathrm{mL}^{-1}$. The content of the silver element in the AgNPs was $3.75 \mu \mathrm{g} \mathrm{mL} \mathrm{m}^{-1}$ and the content of the silver element in ADGDDP and ADG-DDPC were $20.36 \mu \mathrm{g} \mathrm{mL} \mathrm{mL}^{-1}, 20.43 \mu \mathrm{g} \mathrm{mL}^{-1}$, respectively, measured using ICP-MS.

As shown in Fig. 2a, AgNPs have a strong characteristic absorption peak at $400 \mathrm{~nm}$, and the peak shape is narrow and symmetrical. We diluted ADG-DDPC with ultrapure water by half, and as expected, the UV absorbance ratio also decreased by half, and the position of the maximum absorption peak was unchanged (Fig. 2b). After incubation with $200 \mu \mathrm{M} \mathrm{H}_{2} \mathrm{O}_{2}$ in ADG for $40 \mathrm{~min}$, the silver nanoparticles began to "erode" and the originally closely packed GQDs were randomly dispersed (Fig. 2c).

From the UV-VIS absorption curved of AgNPs, it indicates that the AgNPs synthesized using this method are relatively homogeneous, as when the surface has adsorbed DNA, the characteristic absorption peak of the complex is red shifted by $5 \mathrm{~nm}$ and the absorbance decreases, and the peak shape is more asymmetric compared to that of the AgNPs. This is due to the fact that the molecular weight of the DNA molecule $\left(M_{\mathrm{w}}\right.$, $\sim 5303.6 \mathrm{Da})$ is much greater than the molecular weight of the citrate $\left(M_{\mathrm{w}}, \sim 185 \mathrm{Da}\right)$, when the DNA modification is adsorbed onto the surface of the AgNPs, the refractive index of the medium around the AgNPs will increase, and the particle size range is wider than that of the AgNPs. Therefore, the adsorption modification of the DNA molecules on the surface of the AgNPs will cause the red-shift of the characteristic absorption peaks of AgNPs, and the decrease of absorbance may be due to the loss of some AgNPs during the adsorption process. ${ }^{62}$ The change in the UV characteristic peak proves that the DNA is indeed adsorbed onto the surface of the AgNPs. When the ADG cores were coated with asymmetric phospholipids, the UV

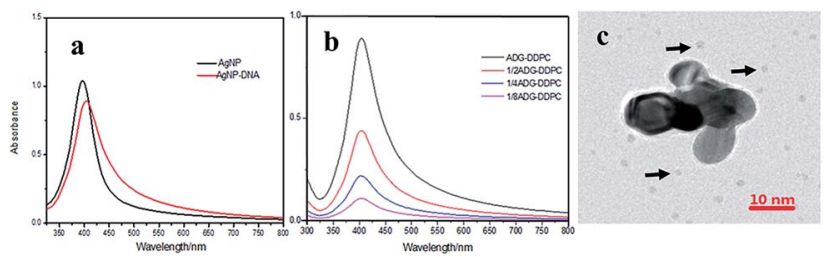

Fig. 2 (a) UV-VIS absorption of AgNPs and AgNP-DNA; (b) UV-VIS absorption of ADG-DDPC diluted with ultrapure water; (c) TEM of the ADG after treating with $200 \mu \mathrm{M} \mathrm{H}_{2} \mathrm{O}_{2}$. The GQDs are indicated by the arrows. absorption peaks were not significantly different from those of the AgNPs-DNA. After diluted with ultrapure water, the UV absorbance of ADG-DDPC was changed according to the dilution without shift of absorption peak, which proved that the internal particle size of the synthesized material was uniform.

When the ADG cores were treated with $\mathrm{H}_{2} \mathrm{O}_{2}$, the silver nanoparticles began to "erode" and the GQDs were unpacked. This underlying mechanism is probably due to the following reaction, shown in formula (2):

$$
\mathrm{Ag}+\mathrm{H}_{2} \mathrm{O}_{2}+\mathrm{H}^{+}=\mathrm{Ag}^{+}+\cdot \mathrm{OH}+\mathrm{H}_{2} \mathrm{O}
$$

It was proven that the AgNPs were corroded and agglomerated by $\mathrm{H}_{2} \mathrm{O}_{2}$, the $\pi-\pi$ bonds of the GQDs and DNA are destroyed, the GQDs are separated from AgNPs, and the fluorescence properties are restored. Using the characteristically high levels of $\mathrm{H}_{2} \mathrm{O}_{2}$ in cancer cells compared to the low $\mathrm{H}_{2} \mathrm{O}_{2}$ content in normal cells, we can use this property for cancer treatment in order to realize the integration of diagnosis and treatment.

The morphology of AgNPs, GQDs and ADG-DDPC were viewed under AFM and TEM. It can be seen from AFM and TEM that the particle size distribution of AgNPs prepared by this method is uniform and the properties are stable (Fig. 3a1a5, enlarged images are shown in Fig. $\mathrm{S} 1 \dagger)$. We can see from the TEM diagram that using the ssDNA as a "bridge" is very necessary (Fig. 3c1-c2, enlarged images are shown in Fig. S3†). Without the addition of DNA, the GQDs are randomly dispersed and do not exist on the surface of the AgNPs. As we can see from the TEM diagram, after adding the DOTAP and the DSPE-PEG2000-cRGD to wrap the ADG kernel, the original random particles are transformed into the smooth particles with larger particle sizes. Using DOTAP as a cationic lipid, the particle size distribution is preferentially adsorbed onto the surface of the DNA with negative charge, thus the first step of encapsulating the core of the phospholipid is realized. In order to enable compound material to remain in vivo for a long cycle, a kind of phospholipid (DSPE-PEG $2000-\mathrm{CRGD}$ ) was added to the outside of DOTAP by changing of the dispersing medium. This also ensures that the materials are less toxic before they reach the target site. Using the Malvern particle sizer measured the particle size of ADG-DDP and ADG-DDPC were found to be $(23.8 \pm 0.5) \mathrm{nm}$ and $(25.2 \pm 0.8) \mathrm{nm}$, respectively. This shows that the addition of cRGD does not have a significant effect on the particle size of the preparation.

The morphology of the particles was detected by AFM and TEM, which indicates that the nucleation process of each particle is relatively stable. In this paper, it is suggested that when DNA is adsorbed onto the surface of AgNPs by using mercapto ssDNA as a "bridge", the refractive coefficient of the AgNPs after adsorption will decrease, and the characteristic absorption peak will be red shifted. The GQDs and ssDNA adsorbed onto the surface of the AgNPs are adsorbed by $\pi-\pi$ bonds. The advantage of this kind of physical adsorption is that it retains the properties of the original material, and the adsorption between substances is much easier compared to that of covalent bonds. Without the addition of DNA (Fig. 3c1 
a
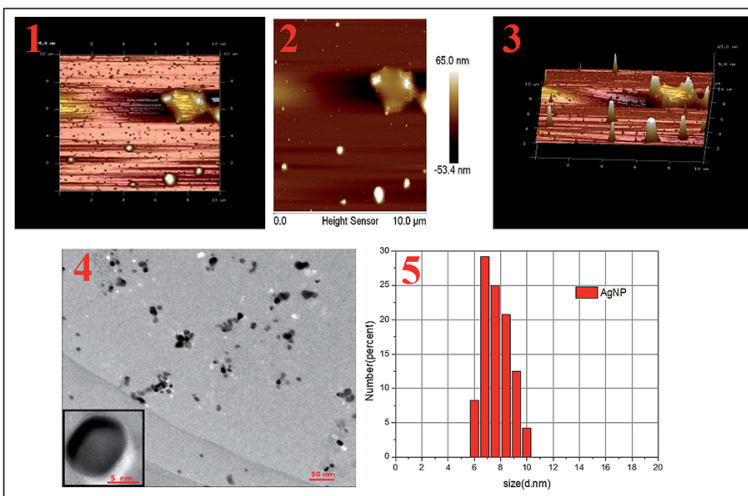

b
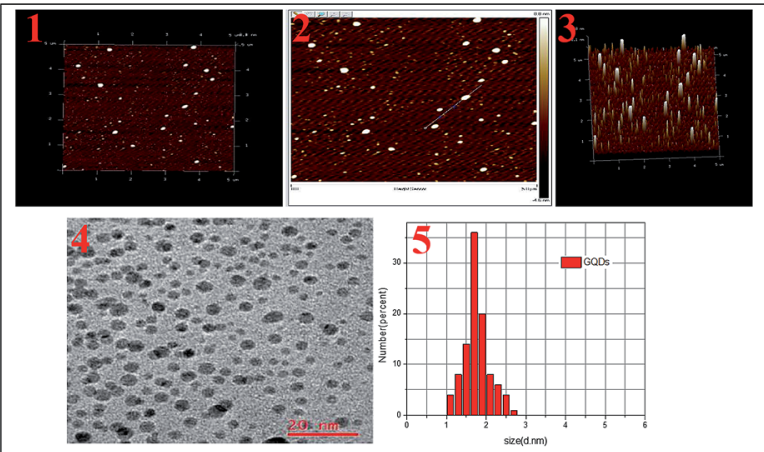

C
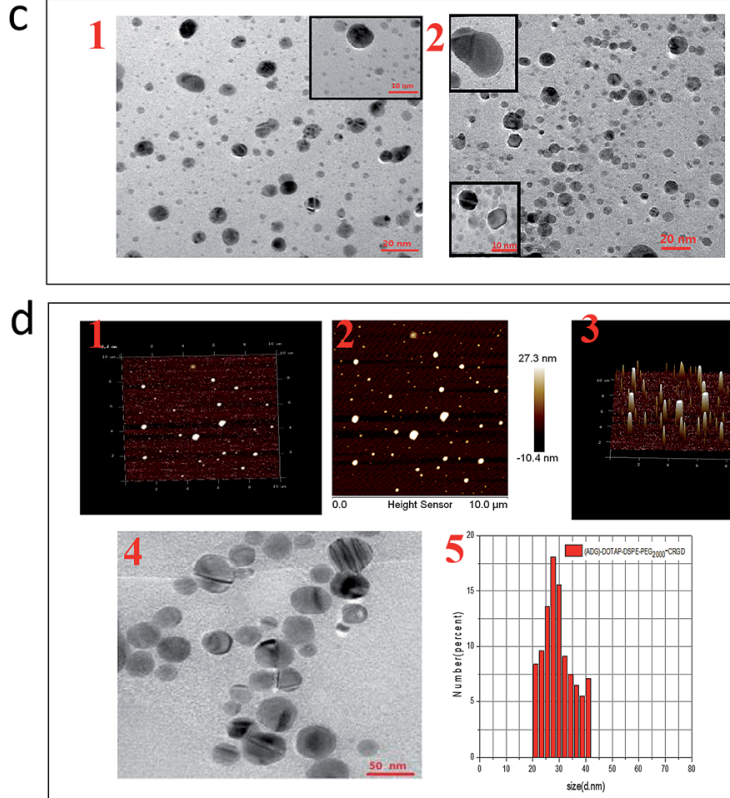

Fig. 3 (a1) Three-dimensional AFM topography image, (a2) AFM height image, (a3) AFM three-dimensional height profile image, (a4) TEM images and (a5) size distribution of AgNPs; (b1) three-dimensional AFM topography image, (b2) AFM height image, (b3) AFM three-dimensional height profile image, (b4) TEM images and (b5) size distribution of GQDs; (c1) TEM images of physical mixture of AgNPs and GQDs and (c2) TEM images of AgNP-DNA-GQDs as squares indicate; (d1) threedimensional AFM topography image, (d2) AFM height image, (d3) AFM three-dimensional height profile image, (d4) TEM image and (d5) size distribution of ADG-DDPC (enlarged images are shown in Fig. S1-S4†).

and c2), the GQDs are randomly dispersed and do not exist on the surface of the AgNPs, which affirmed the necessary existence of SsDNA in ADG.

\section{Stability of ADDG, ADG-DDP and ADG-DDPC}

It is not difficult to see from Table 1 that with the prolongation of time, the phospholipid-coated ADG core is prone to aggregation, and the particle size has exceeded $100 \mathrm{~nm}$ at $7 \mathrm{~h}$, while the particle size of ADG-DDP and ADG-DDPC did not change significantly within $48 \mathrm{~h}$. This is because the ADG core is separated by phospholipids, demonstrating that the phospholipid system is stably dispersed in aqueous solution over a period of time.

\section{Fluorescence detection of $\mathrm{H}_{2} \mathrm{O}_{2}$}

To detect $\mathrm{H}_{2} \mathrm{O}_{2}$, aliquots $(400 \mu \mathrm{L})$ of $\mathrm{BR}$ buffer $(\mathrm{pH}=6)$ containing $200 \mathrm{pM}$ ADG were spiked with $\mathrm{H}_{2} \mathrm{O}_{2}(0-400 \mu \mathrm{M})$ and reacted at $37{ }^{\circ} \mathrm{C}$ for $40 \mathrm{~min}$. Then, the fluorescence was measured under excitation at $350 \mathrm{~nm}$. Fig. 4a shows the emission spectra of GQDs at different excitation wavelengths. It can be seen from the figure that the maximum emission wavelength of the GQDs is $425 \mathrm{~nm}$ under excitation light of $350 \mathrm{~nm}$. Fig. 4b shows the fluorescent spectra of ADG in the presence of variable concentrations of $\mathrm{H}_{2} \mathrm{O}_{2}$. The ADG nanocomposite exhibited weak fluorescence in the absence of $\mathrm{H}_{2} \mathrm{O}_{2}$. However, the fluorescence intensity around $425 \mathrm{~nm}$ gradually enhanced upon increasing $\mathrm{H}_{2} \mathrm{O}_{2}$ addition. A good linearity concentration was raised from 2.0 to $400 \mu \mathrm{M}$, deriving a detection limit of $0.2 \mu \mathrm{M}$. The results indicate that our constructed ADG nanocomposite is appropriate for highly sensitive quantification of $\mathrm{H}_{2} \mathrm{O}_{2}$ in aqueous solution.

\section{Cell viability and LDH activity}

Different concentrations of AgNPs and GQDs dispersions were added to HeLa cells and incubated for 24 h. As shown in Table 2, the AgNPs showed a strong toxicity in HeLa cells with an $\mathrm{IC}_{50}$ value of $347.78 \mathrm{ng} \mathrm{mL} \mathrm{m}^{-1}$, while GQDs showed no cytotoxicity below $150 \mu \mathrm{g} \mathrm{mL} \mathrm{m}^{-1}$. This is consistent with what we expected to achieve. Both the ADG-DDP and ADG-DDPC had the effect of killing the HeLa cells in a certain concentration range, and the killing effect was dose-dependent.

Upon addition of DSPE-PEG 2000 -cRGD, ADG-DDPC exhibited a lower $\mathrm{IC}_{50}$ compared to that of ADG-DDP, which was consistent with the results of the LDH activity of the ADG-DDP- or ADG-DDPC-treated group (Fig. 5). In order to allow the rapid growth and reproduction of tumors, tumor cells often have a high glucose uptake and possess the ability to convert glucose into lactic acid. ${ }^{63}$ LDH is a key enzyme in glycolysis, which catalyzes the terminal steps of anaerobic glycolysis to deoxidize pyruvic acid by catalyzing the reversible reaction between pyruvic acid and lactic acid, with this $\mathrm{NAD}^{+} / \mathrm{NADH}$ interconversion. ${ }^{64}$ The activity of LDH can be used as a marker of the level of glycolysis level. The activity of LDH was calculated after equal amounts of ADG-DDP and ADG-DDPC were incubated in the cells for $24 \mathrm{~h}$ at $37^{\circ} \mathrm{C}$ in $5 \% \mathrm{CO}_{2}$. At the same dose, the intracellular LDH activity of the ADG-DDPC group was significantly lower than that of the ADG-DDP group, indicating that the glycolytic pathway of the cells was significantly inhibited after adding ADG-DDPC. 
Table 1 Particle size of ADG, ADG-DDP and ADG-DDPC within 48 hours in the absence of light $4{ }^{\circ} \mathrm{C}$

\begin{tabular}{|c|c|c|c|c|c|c|c|c|c|c|}
\hline & 0 & 1 & 2 & 3 & 5 & 7 & 10 & 12 & 24 & 48 \\
\hline Size/nm & 18.78 & 23.3 & 29.93 & 53.61 & 89.12 & 104.4 & 352.2 & 378.4 & 594.9 & 832 \\
\hline PDI & 0.332 & 0.371 & 0.516 & 0.310 & 0.521 & 0.621 & 0.519 & 0.421 & 0.668 & 0.877 \\
\hline PDI & 0.025 & 0.013 & 0.032 & 0.132 & 0.142 & 0.035 & 0.120 & 0.113 & 0.032 & 0.246 \\
\hline Size/nm & 25.18 & 25.18 & 25.32 & 25.34 & 25.38 & 25.38 & 26.10 & 26.21 & 27.63 & 30.33 \\
\hline PDI & 0.035 & 0.071 & 0.121 & 0.214 & 0.105 & 0.213 & 0.189 & 0.233 & 0.310 & 0.279 \\
\hline
\end{tabular}
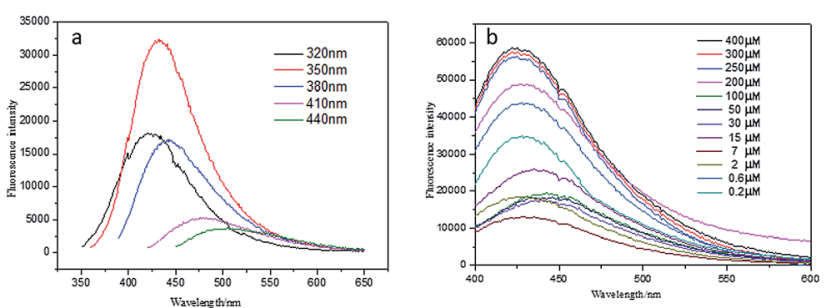

Fig. 4 (a) Emission spectra of $\mathrm{NH}_{2}$-GQDs at different excitation wavelengths. (b) Fluorescence spectra of ADG as functions of different concentrations of $\mathrm{H}_{2} \mathrm{O}_{2}$. The signal changes as $\mathrm{H}_{2} \mathrm{O}_{2}$ concentrations increase $(0.6-400 \mu \mathrm{M})$.

Table 2 The $\mathrm{IC}_{50}$ values of each material and preparation $(n=6)$

\begin{tabular}{ll}
\hline Preparations & $\mathrm{IC}_{50}\left(\mathrm{ng} \mathrm{\textrm {mL } ^ { - 1 }}\right)$ \\
\hline GQDs & $<150 \times 10^{3}$ \\
AgNPs & $347.78 \pm 0.06$ \\
ADG-DDP & $270.85 \pm 0.07$ \\
ADG-DDPC & $209.68 \pm 0.09$
\end{tabular}

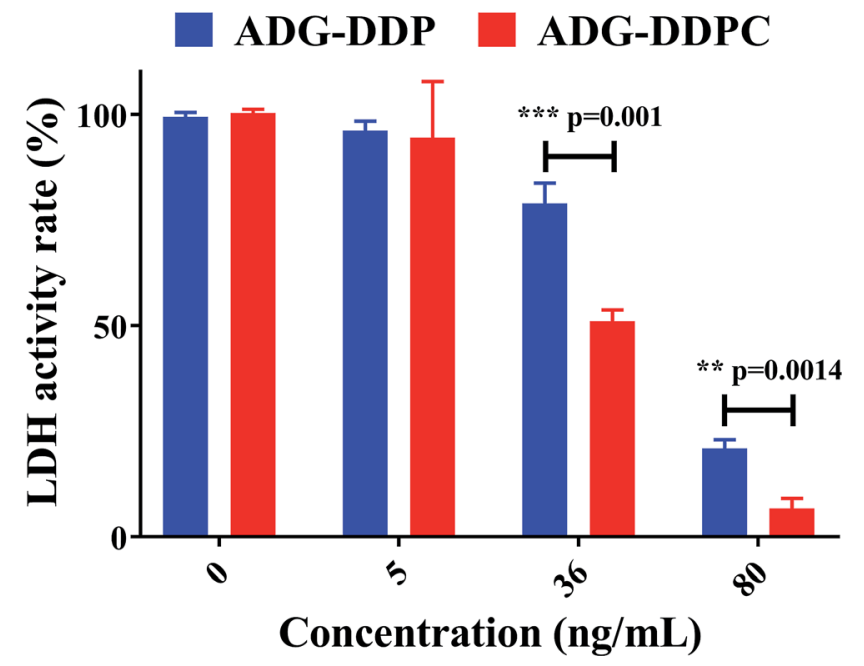

Fig. 5 LDH activity of the HeLa cells after being incubated with ADGDDP or ADG-DDPC.

It has been found that nano-silver has a strong cytotoxicity against many cancer cells, which proves that AgNPs have a realistic basis as an anti-tumor preparation. However, there is no definitive conclusion as to how nanosilver produces cancer cell toxicity. Based on this characteristic, AgNPs and $\mathrm{H}_{2} \mathrm{O}_{2}$ could react to release highly toxic silver ions in the tumor cells, and the released silver ions could bind to enzymes in the respiratory chain, inhibit the synthesis of adenosine triphosphate. In addition, the generated silver ions could also bind to the base pair of DNA generated within the cell, inhibiting DNA replication and amplification, and blocking the most essential part of cell proliferation.

\section{Cell uptake}

ADG-DDP and ADG-DDPC (24 $\mathrm{ng} \mathrm{mL}^{-1}$ ) were added to HeLa cells and incubated for $6,12 \mathrm{~h}$ at $37^{\circ} \mathrm{C}$ in $5 \% \mathrm{CO}_{2}$, and GQDs in both phospholipids were emitted. Intensity of fluorescence, and with the prolongation of incubation time, the intensities of ADG-DDPC were more obvious, cRGD-added phospholipid material more easily into the cell. On the one hand, due to the presence of $\mathrm{H}_{2} \mathrm{O}_{2}$ in tumor cell, the DNA in the phospholipid material is "eroded", releasing fluorescent quenched GQDs and restoring its fluorescence, this result is consistent with expectations. With the prolongation of incubation time, the fluorescence intensities of ADG-DDPC were more obvious (Fig. 6a and b, enlarged images are shown in Fig. S5 $\dagger$ ). The percentages of cells exhibiting fluorescence were indicated by the fluorescence of released GQDs in cells and quantified in Fig. 6c. The results indicated that in the safe dose range, cRGD-modified nanoparticles were more easily taken up into these cells and the ADG cores were unpacked to release GQDs, which was consistent with the lower $\mathrm{IC}_{50}$ and $\mathrm{LDH}$ activity measured in the ADGDDPC group in Table 2 and Fig. 5.

From the cell uptake experiments, we can see that the intracellular fluorescence intensity of the nanomaterials containing DSPE-PEG ${ }_{2000}$-cRGD is more obvious when compared with that of the ADG-DDP, that is, the luminescence efficiency of the GQDs is higher. This may be due to the fact that the nanomaterials containing the transmembrane peptide cRGD can enter the tumor cells more quickly and therefore the fluorescence recovery of the GQDs is faster. ${ }^{65}$

HeLa cells were incubated with ADG-DDP or ADG-DDPC for $6 \mathrm{~h}$ and $12 \mathrm{~h}$ individually and it was observed that ADG-DDPC was more easily uptaken by cells and could release GQDs from ADG under $\mathrm{H}_{2} \mathrm{O}_{2}$. Because tumor cells metabolize much faster than normal cells, the amount of hydrogen peroxide produced in the cells is also higher than that in normal cells. It is reported that the content of $\mathrm{H}_{2} \mathrm{O}_{2}$ in tumor cells can reach 10$50 \mu \mathrm{M} .{ }^{66}$ Based on this feature, AgNPs and $\mathrm{H}_{2} \mathrm{O}_{2}$ after the following reaction occurs in the tumor cells, the highly toxic 


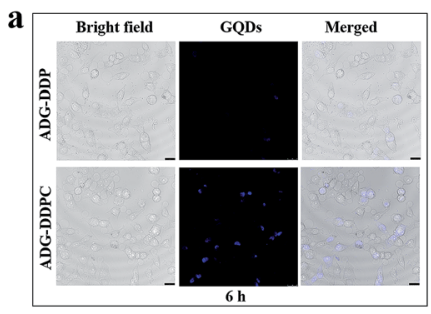

c
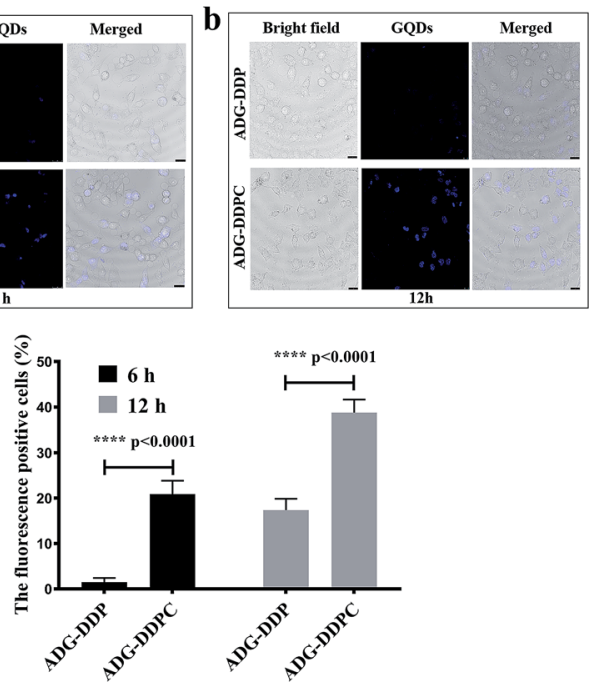

Fig. 6 Fluorescence micrographs of HeLa cells incubated with ADGDDP and ADG-DDPC for $6 \mathrm{~h}$ (a) and $12 \mathrm{~h}$ (b). From the left are bright field, fluorescence from GQDs (blue light) and merged images. Bar equals $30 \mu \mathrm{m}$; (c) the quantification of the percentages of fluorescence positive cells (fluorescence caused by released GQDs) after incubating with ADG-DDP or ADG-DDPC for 6 and $12 \mathrm{~h}$.

silver ions are released, and the released silver ions can bind to the enzymes in the respiratory chain, uncoupling the respiratory chain, inhibiting the synthesis of adenosine triphosphate (ATP), thereby making the cancer cells cannot proliferate normally. In the presence of the target $\mathrm{H}_{2} \mathrm{O}_{2}, \mathrm{H}_{2} \mathrm{O}_{2}$ corrodes the silver nanoparticles, reduces the absorption intensity of the silver nanoparticles, and weakens the energy transfer between the AgNPs and the GQDs, thereby recovering the fluorescence of the GQDs.

\section{Anti-tumor growth effect}

Based on the favorable capability of inducing apoptosis, an in vivo therapeutic efficacy study was performed to assess the potentials of ADG-DDP or ADG-DDPC for inhibiting tumor growth. It can be seen that ADG-DDP, ADG-DDPC and PTX injection could suppress tumor growth (Fig. 7a) and the average of tumor growth inhibition rates for ADG-DDP, ADG-DDPC and PTX Injection were calculated to be $24.8,50.9$, and $57.7 \%$,
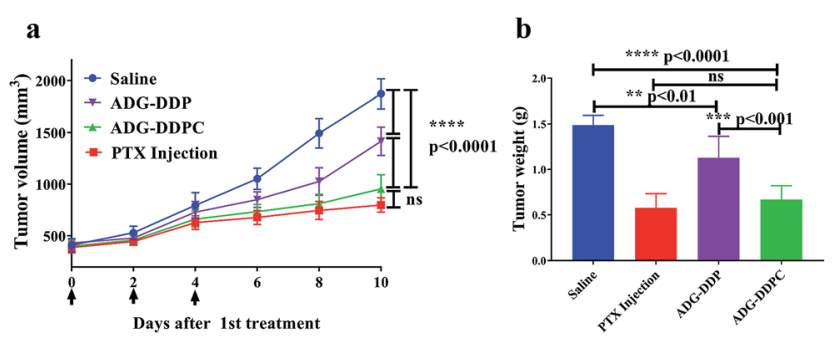

Fig. 7 (a) The tumor growth curve of mice treated with ADG-DDP, ADG-DDPC and PTX injection intravenously, and the mice were intravenously injected with saline as a control. Arrows indicate the days for injections; (b) tumor weights of mice in different groups ( $n=6)$. respectively, according to the tumor weight (Fig. 7b). As the high toxicity of anticancer drugs usually leads to a reduction in body weight and necrosis in organs, the body weight of the PTX Injection group showed a slightly decreasing trend, whereas little effect on body weight was observed in the ADG-DDPC group (data not shown).

Taking all of these results into consideration, ADG-DDPC, as a new multifunctional nano-preparation, has a good effect in inhibiting tumors and is less toxic than commercially available PTX Injections. In the novel ADG-DDPC nanoparticle preparations, each component could contribute to some degree to the outstanding efficacy on tumor inhibition. Targeted phospholipids can circulate in mice tumor for a prolonged time. When cRGD recognizes tumor cells and binds to the tumor cell surface receptors, the nanomaterials are brought into the cells by endocytosis. Owing to the higher concentration of $\mathrm{H}_{2} \mathrm{O}_{2}$ inside the tumor cells, the AgNPs entering the cell are dissolved, and the silver ions are released, When the AgNPs are etched, GQDs are released, thereby achieving the purpose of the design, that is, antitumor property.

\section{Tissue analysis in $B A L B / c$ nude mice}

H\&E staining of the PBS, GQDs, ADG-DDP and ADG-DDPC groups did not show obvious kidney injury, pulmonary toxicity, cardiac damage, or inflammatory infiltrates in the spleen (Fig. 8). The control tumor had many mitotic figures,

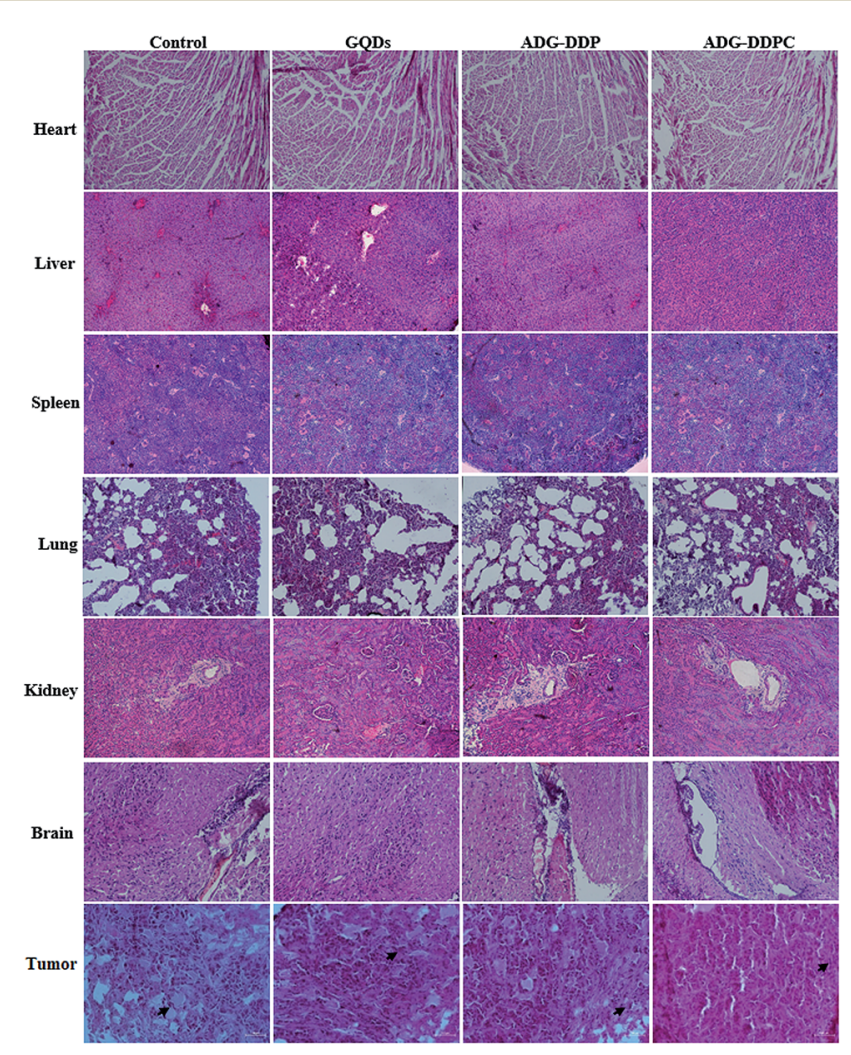

Fig. 8 HEE staining of major organs $(10 x)$ and tumors $(20 x)$ of groups treated with GQDs, ADG-DDP and ADG-DDPC groups. Arrows indicate the presence of spindle-shaped cells. 


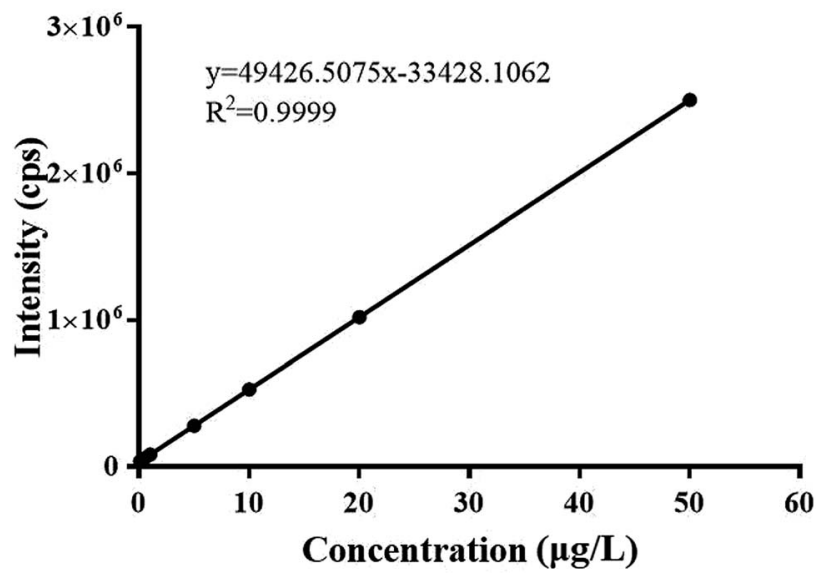

Fig. 9 Standard curve of silver elemental solution.

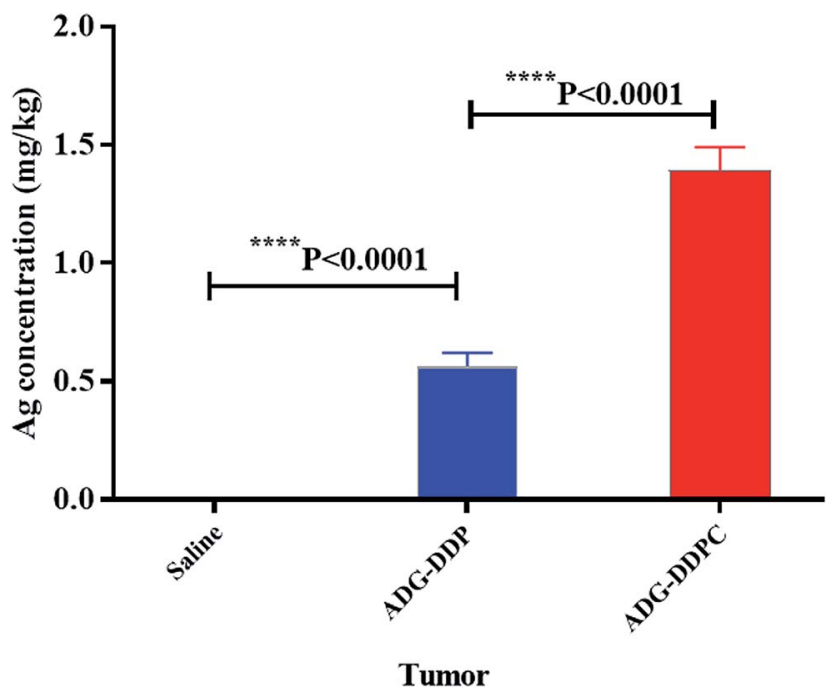

Fig. 10 Silver content in ADG-DDP and ADG-DDPC groups of tumor tissue.

showing the high mitotic activity of tumor cells. ${ }^{67}$ Tumors that were treated with ADG-DDPC experienced a dramatic decrease in the number of mitotic figures, and exhibited more basophilic and uniform nuclei.

The standard curve of silver elemental solution are shown in the Fig. 9, the correlation coefficient is equal to 0.9999, indicating that silver elemental have a good linear relationship.

The results of silver content determination in the tumor are shown in the Fig. 10. Silver was not detected in the saline group, and the intratumoral silver content in the ADG-DDPC group was significantly higher than that in the ADG-DDP group, indicating that the cRGD could be better transported the nanosilver into the tumor.

\section{Conclusions}

Using the ssDNA as a "bridge", AgNPs were connected to nontoxic luminescent materials GQDs to form a core, which were encapsulated by non-toxic hybrid phospholipid membrane which have a long circulation time in vivo and the ability of tumor targeting. This study presented a novel nanometer preparation of ADG-DDPC that can inhibit the growth of tumor cells and emit light within tumor cells.

\section{Conflicts of interest}

There are no conflicts to declare.

\section{Acknowledgements}

The authors thank the National Natural Science Fund of China (81560575, 81760639, 81603054 and 81373347), the Project funded by Jiangxi Province Postdoctoral Science Foundation (2016KY08), the Natural Science Fund of Jiangxi Province (20171BAB215066, 20171ACB21074), the Jiangxi Province Outstanding Young Talents Program (20162BCB23034, 20171BCB23097), Young Jinggang Scholar of Jiangxi Province (Jing Zhang), “1050” Youth Talent Project of Jiangxi University of Traditional Chinese Medicine (Jing Zhang), Jiangxi BaiQianWan Talents Program (Xiang Li), the Project funded by China Postdoctoral Science Foundation (2016M602084), the Project funded by Jiangxi Provincial Health and Family Planning Commission (2016A008), the Fund for First-Rate Discipline of Chinese Materia Medica (JXSYLXK-ZHYAO055, JXSYLXK-ZHYAO056, JXSYLXK-ZHYAO019) and the Youth Foundation of Jiangxi Provincial Education Department (GJJ170748) for financial support.

\section{References}

1 H. Long, G. Li, X. Wen, M. Zhang, Y. Wei, W. Xie, P. Zhong and L. Pang, Gene, 2018, 678, 270-279.

2 S. Conti, A. Vexler, L. Hagoel, L. Kalich-Philosoph, B. W. Corn, N. Honig, N. Shtraus, Y. Meir, I. Ron, I. Eliaz and S. Lev-Ari, Integr. Cancer Ther., 2018, 17, 1225-1234.

3 M. Thomsen and L. Vitetta, Integr. Cancer Ther., 2018, 17, 1027-1047.

4 P. Thankamony, R. Chandar, J. Kattoor and R. K. Nair, J. Pediatr. Adol. Gynec., 2018, 31, 629-631.

5 Y. Wang, Q. Lei, Z. Yan, C. J. Shen and N. Wang, Biochem. Pharmacol., 2018, 155, 510-523.

6 S. Liu, W. J. Song, F. S. Liu, J. W. Zhang and S. Q. Zhu, Exp. Eye Res., 2018, 172, 144-151.

7 J. Y. Lee, S. J. Chung, H. J. Cho and D. D. Kim, Biomaterials, 2016, 85, 218-231.

8 M. Green, J. Cunningham, D. O'Connell and G. Garvey, Aust. Health Rev., 2017, 41, 231-233.

9 Y. Song, W. Li, S. Y. Meng, W. Zhou, B. Su, L. Tang, Y. M. Zhao, X. Y. Wu, D. Z. Yin, M. X. Fan and C. C. Zhou, Nanoscale Res. Lett., 2018, 13, 1-9.

10 W. P. He, J. Frueh, Z. W. Wu and Q. He, Langmuir, 2016, 32, 3637-3644.

11 I. K. Kyriakou, K. Mavridis, D. P. Kalogianni, T. K. Christopoulos, P. C. Ioannou and A. Scorilas, Anal. Bioanal. Chem., 2018, 410, 971-980. 
12 N. Dong, M. Y. Dong, A. T. Zhao, Q. J. Zhu, Z. Tao and Y. Zhao, Sci. China: Chem., 2010, 53, 2304-2310.

13 M. F. Dias, B. C. P. Figueiredo, J. Teixeira-Neto, M. C. A. Guerra, S. L. Fialho and C. A. Silva, Biomed. Pharmacother., 2018, 103, 1107-1114.

14 E. M. Mastria, L. Y. Cai, M. J. Kan, X. H. Li, J. L. Schaal, S. Fiering, M. D. Gunn, M. W. Dewhirst, S. K. Nair and A. Chilkoti, J. Controlled Release, 2018, 269, 364-373.

15 Z. S. Qian, X. Y. Shan and L. J. Chai, J. Nanoscale., 2014, 6, 5671-5674.

16 O. V. Sedelnikova, C. P. Ewels, L. G. Bulusheva and A. V. Okotrub, J. Struct. Chem., 2018, 59, 870-876.

17 B. Oh and C. H. Lee, Pharm. Res., 2016, 33, 2736-2747.

18 P. Huang, J. J. Shi, M. Zhang, X. H. Jiang, H. X. Zhong, Y. M. Ding, X. Cao, M. Wu and J. Lu, J. Phys. Chem. Lett., 2016, 7, 2888-2892.

19 G. Battogtokh and Y. Tag, J. Controlled Release, 2016, 234, 1020.

20 Z. S. Qian, X. Y. Shan, L. J. Chai, J. J. Ma, J. R. Chen and H. Feng, Biosens. Bioelectron., 2014, 60, 64-70.

21 M. A. Ribas, A. K. Singh, P. B. Sorokin and B. I. Yakobson, Nano Res., 2011, 4, 143-152.

22 H. Hu, L. Xiong, J. Zhou, F. Y. Li, T. Y. Cao, C. H. Huang, et al., J. Chemistry., 2009, 15, 3577.

23 J. S. Klein, G. S. Mitchell and S. R. Cherry, Phys. Med. Biol., 2017, 62, 4183.

24 F. Musumeci, L. A. Applegate, G. Privitera, A. Scordino, S. Tudisco and H. Niggli, J. Photochem. Photobiol., B, 2005, 79, 93-99.

25 B. M. Seddon, D. J. Honess, B. Vojnovic, G. M. Tozer and P. Workman, J. Radiat. Res., 2001, 155, 837.

26 B. P. Aduev, G. M. Belokurov, D. R. Nurmukhametov and N. V. Nelyubina, Combust., Explos. Shock Waves, 2012, 48, 361-366.

27 X. L. Cao, M. Tang, F. Liu, Y. Y. Nie and C. S. Zhao, Colloids Surf., B, 2010, 81, 555-562.

28 L. Deng, X. Y. Ouyang, J. Y. Jin, C. Ma, Y. Jiang, J. Zheng, J. S. Li, Y. H. Li, W. H. Tan and R. H. Yang, Anal. Chem., 2013, 85, 8594-8600.

29 A. Karolak, V. C. Estrella, A. S. Huynh, T. G. Chen, J. Vagner, D. L. Morse and K. A. Rejniak, Sci. Rep., 2018, 8, 36-38.

30 J. K. Patra and K. H. Baek, Int. J. Nanomed., 2016, 11, 46914705.

31 D. C. Emerson, D. F. Luiz Francisco Poli, P. O. José, M. S. Marcelo, D. O. Roberto Angelo, F. F. Fabiane, S. M. Valeria, Z. Valtencir, S. L. Anna Sara and F. C. Silvia, J. Nanobiotechnol., 2015, 13, 64.

32 V. Singh and S. Ahmad, Cellulose, 2012, 19, 1759-1769.

33 M. R. C. Sytu and D. H. Camacho, J. Bionanosci., 2018, 8, 835844.

34 X. Chen and H. J. Schluesener, Toxicol. Lett., 2008, 176, 1-12.

35 O. Choi and Z. Hu, Environ. Sci. Technol., 2008, 42, 45834588.

36 R. K. Thapa, J. H. Kim, J. H. Jeong, J. H. Jeong, B. S. Shin, H. G. Choi, C. S. Yong and J. O. Kim, Colloids Surf., B, 2017, 153, 95-103.
37 D. Sandhanasamy, A. Mohamad, V. Radhakrishnan, A. Akram, A. Nehad, A. Musaad, M. Kadarkarai, S. Shaban, N. Marcello and B. Giovanni, J. Cluster Sci., 2017, 28, 595605.

38 C. Pimentel, R. Guadalupe, R. B. Viviana, M. M. S. Eduardo, G. G. Consuelo and H. Juan Paulo, J. Biomater. Sci., Polym. Ed., 2016, 27, 218-234.

39 S. A. Sadat Shandiz, A. M. Shafiee, D. Shahbazzadeh, A. Assadi, C. R. Ahangari, V. Asgary and S. Salehi, Artif. Cells, Nanomed., Biotechnol., 2017, 45, 1-10.

40 R. G. Saratale, H. S. Shin, G. Kumar, G. Benelli, D. S. Kim and G. D. Saratale, Artif. Cells, Nanomed., Biotechnol., 2018, 46, 211-222.

41 Z. Ge, Q. Chen, K. Osada, X. Liu, T. A. Tockary, S. Uchida, A. Dirisala, T. Ishii, T. Nomoto, K. Toh, Y. Matsumoto, M. Oba, M. R. Kano, K. Itaka and K. Kataoka, Biomaterials, 2014, 35, 3416-3426.

42 M. K. Hossain, H. Y. Cho, K. J. Kim and J. W. Choi, Sci. Adv. Mater., 2014, 6, 2491-2495.

43 J. A. Jacob and A. Shanmugam, Colloids Surf., B, 2015, 136, 1011-1016.

44 N. Miura and Y. Shinohara, Biochem. Biophys. Res. Commun., 2009, 390, 733-737.

45 M. Jha, N. G. Shimpi and S. S. Sonawane, J. Genet. Eng. Biotechnol., 2018, 16, 115-124.

46 H. Y. Xu, F. Qu, H. Xu, W. H. Lai, Y. A. Wang, Z. P. Aguilar and H. Wei, BioMetals, 2012, 25, 45-53.

47 A. Ivask, O. Bondarenko and N. Jepihhina N, Anal. Bioanal. Chem., 2010, 398, 701-716.

48 D. Nandita and R. Chidambaram, Environ. Chem. Lett., 2016, 14, 477-485.

49 A. R. Lee, S. J. Lee, M. Lee, M. Y. Nam, S. Lee, J. Choi, H. J. Lee, D. U. Kim and K. L. Hoe, Toxicol. Sci., 2018, 161, 171-185.

50 A. Rinna, Z. Magdolenova, A. Hudecova, M. Kruszewski, M. Refsnes and M. Dusinska, Mutagenesis, 2015, 30, 59-66.

51 C. A. Verónica, A. Sungeun, Y. S. Shakina, W. Chao, M. Ramya and C. Y. Deok, In Vitro Cell. Dev. Biol.: Anim., 2016, 52, 1012-1019.

52 H. Margarita, R. Gonzalo, J. M. P. Raul, V. G. R. Jose, D. Concepcion and S. Paz, Nanoscale Res. Lett., 2012, 7, 364.

53 D. W. Guo, D. D. Dou, L. Ge, Z. H. Huang, L. P. Wang and N. Gu, Colloids Surf., B, 2015, 134, 229-234.

54 Z. Huang, F. Pu, Y. Lin, J. Ren and X. Qu, Chem. Commun., 2011, 47, 3487-3489.

55 T. Hada and Y. Sakurai, Pharmaceutics, 2015, 7, 320-333.

56 B. Dicheva, T. Hagen, A. Seynhaeve, M. Amin, A. Eggermont and G. Koning, Pharm. Res., 2015, 32, 3862-3876.

57 S. He, B. Cen, L. Liao, Z. Wang, Y. X. Qin, Z. M. Wu, W. J. Liao, Z. Y. Zhang and A. M. Ji, Drug Delivery, 2017, 24, 471-481.

58 J. Hedhli, S. L. L. Slania, A. Pska, A. Czerwinski, C. J. Konopka, M. Wozniak, M. Banach, I. T. Dobrucki, L. Kalinowski and L. W. Dobrucki, Sci. Rep., 2018, 8, 5401.

59 G. B. Braun, K. N. Sugahara, O. M. Yu, V. R. Kotamraju, T. Molder, A. M. Lowy, E. Ruoslahti and T. Teesalu, J. Controlled Release, 2016, 232, 188-195. 
60 P. F. Liu, L. B. Qin, Q. Wang, Y. Sun, M. J. Zhu, M. Shen and Y. R. Duan, Biomaterials, 2012, 33, 6739.

61 M. Amin, A. Badiee and M. R. Jaafari, Int. J. Pharm., 2013, 458, 324-333.

62 X. Zhang, M. R. Servos and J. W. Liu, Chem. Commun., 2012, 48, 10114-10116.

63 Z. Guo, J. Jia, M. Yao, J. Kang, Y. Wang, X. Yan, L. Zhang, Q. Lv, X. Chen and F. Lu, Exp. Cell Res., 2018, 373, 211-220. 64 L. Yang, D. Wang, X. T. Wang, Y. P. Lu and L. Zhu, Biochem. Biophs. Res. Commun., 2018, 507, 128-135.
65 C. D. Jorge, V. G. Juan, H. M. Fernando, C. S. Lionel, L. S. Juan, N. V. Juanita, M. H. Francisco, K. P. Rodrigo, C. F. Clemencia, B. G. Margarita, R. E. Ivonne and F. O. Maritza, Rev. Chil. Obstet. Ginecol., 2008, 73, 308-312. 66 Z. Wu, Y. Wang, X. Meng, X. Wang, Z. Li, S. Qian, Y. Wei, L. Shu, Y. Ding, P. Wang and Y. Peng, Int. J. Mol. Med., 2018, 42, 3157-3170.

67 A. Goel and H. Goel, J. Indian Soc. Pedod. Prev. Dent., 2011, 29, S61-S65. 